\title{
RENTABILIDAD DE LA ECOGRAFÍA EN UN SERVICIO DE UROLOGÍA.
}

\author{
Miguel Ángel Nogueras Gimeno, María Cruz Marrón Penón, Carlos Guinda Sevillano y \\ Ricardo Espuela Orgaz.
}

Servicio de Urología. Hospital Santa Bárbara. Soria. España.

\begin{abstract}
Resumen.- La ecografía ha demostrado ser una prueba diagnóstica de gran sensibilidad, especificidad y exactitud. El urólogo es el profesional mas capacitado para sacar el máximo rendimiento a esta técnica, de forma que la disponibilidad de ecógrafos por parte de los Servicios de Urología racionaliza y limita la demanda de ecografías, disminuye de forma importante la petición de exploraciones radiológicas y reduce el número de cistoscopias, siendo un elemento indispensable en las consultas de Alta Resolución.
\end{abstract}

Presentamos la estadística sobre la utilización de la ecografía en nuestro Servicio, en el que ha desaparecido la demanda de ecografías desde las Consultas de Urología al Servicio de Radiodiagnóstico. La tasa de utilización es muy alta y refleja el criterio funcional de uso de los ecógrafos.

Miguel Angel Nogueras Gimeno C/Almazán, 15, 3ํㅡ 42004 Soria (España) miguelnogueras@yahoo.es
Al analizar la rentabilidad económica nos hemos encontrado con la ausencia casi total de ensayos clínicos controlados que comparen desde el punto de vista de rentabilidad económica las distintas técnicas de imagen utilizadas en Urología. No existen estudios de costes que comparen las ecografías realizadas por urólogos con las ecografías solicitadas a los Servicios de Radiología.

Palabras clave: Ecografía. Rendimiento. Eficacia. Tasa de utilización Coste-beneficio. Variabilidad. Ecografía urológica.

Summary.- Ultrasonography has demonstrated to be a precise test with high sensitivity and specificity. The urologist is the most capable professional to obtain the maximum effectiveness of this technique, so that the availability of ultrasound machines in urology departments rationalizes and limits the demand of ultrasound tests, diminishes significantly the indication of radiological tests and the number of cystoscopies, being an indispensable element in high-resolution office consultations.

We report the statistics of the use of ultrasound in our department, with the disappearance of the demand of ultrasound tests from the urology outpatient clinic to the radiology department. The use rate is high, reflecting the functional criteria for use of the ultrasound equipment. In the economic analysis we found there is an almost complete absence of controlled clinical trials comparing the cost effectiveness of imaging tests in urology. There are not cost studies comparing ultrasounds performed by the urologist with those performed by radiologists.

Keywords: Ultrasound. Cost effectiveness. Efficacy. Use rate. Cost benefit. Variability. Urological ultrasound. 


\section{INTRODUCCIÓN}

La ecografía constituye la aportación más importante de los últimos 20 años al diagnóstico urológico. Se considera en la actualidad como la técnica inicial de diagnóstico por imagen en gran número de patologías urológicas, y un método ideal para el seguimiento de los pacientes. Es la técnica de imagen más extendida y los actuales ecógrafos nos proporcionan imágenes del riñón, vejiga, próstata, pene y escroto que eran impensables hace unos años. La ecografía es una técnica de alta sensibilidad, segura, inocua, rápida y de bajo coste.

La urología es una especialidad en que las técnicas de diagnóstico por imagen son imprescindibles y por ello es lógico que la ecografía se haya incorporado directamente a la especialidad y su utilización se realice de forma directa por el urólogo. Hoy día, se admite que la utilización de los ecógrafos, además de la especialidad de Radiología, se realice por especialidades de diversas áreas quirúrgicas y médicas, que dispongan de la adecuada formación y experiencia. Esta tendencia a la utilización de la ecografía más allá de los Servicios de Radiología ha sido reforzada por la aparición de equipos más baratos, de dimensiones más reducidas y mayor movilidad. Actualmente, los ecógrafos urológicos son muy versátiles, ya que los equipos pueden trasladarse con facilidad al gabinete de exploración o de pruebas especiales, a la consulta, a la cabecera de la cama del paciente hospitalizado o al quirófano. Esta versatilidad es esencial para responder a los retos que nos presenta la ecografía urológica, tanto en su aspecto diagnóstico como en la ecografía intervencionista.

\section{RENTABILIDAD EN EL DIAGNÓSTICO}

Al valorar las aportaciones de la ecografía urológica nos referimos a las tecnologías e indicaciones plenamente aceptadas y difundidas ya sean a nivel diagnóstico o intervencionista, sin entrar en las nuevas tecnologías que están en periodo de implantación y difusión.

Existen magnificas revisiones de conjunto que recogen las indicaciones actuales de la ecografía diagnóstica como la de Passas, J. (1) y McAchran (2), o como la de Rodríguez-Patrón, R (3) sobre biopsias prostáticas ecodirigidas. Por ello, nuestro interés se dirige al análisis del rendimiento que podemos esperar al aplicar esta prueba diagnóstica.

Al valorar la ecografía como prueba instrumental diagnóstica debemos determinar el grado en que la ecografía puede distinguir entre individuos que presentan una patología urológica y los que no la padecen. Ello implica un razonamiento de tipo probabilístico que se expresa en parámetros como sensibilidad, especificidad, valores predictivos, exactitud o índice de correlación de Pearson que son los que caracterizan y permiten valorar una prueba diagnóstica. Sería deseable disponer de estos parámetros en la aplicación de la ecografía a cada una de las patologías urológicas y situaciones clínicas, pero la revisión de la literatura sobre el tema nos indica que todavía carecemos de muchas de estas informaciones. No obstante, en la Tabla I recogemos la rentabilidad de la ecografía urológica en procesos urológicos comunes que requieren de exploraciones por imagen. En esta revisión realizada siguiendo un criterio histórico hemos recuperado aquellos trabajos que consideramos de mayor relevancia, especialmente porque cuantificaban la eficacia diagnóstica de la ecografía, ya que la mayoría de las publicaciones son trabajos de tipo observacional sin análisis estadístico sobre su eficacia.

Desde la introducción de la escala de grises, la ecografía ha demostrado una gran sensibilidad, especificad y exactitud como prueba diagnóstica. Únicamente ha defraudado las expectativas depositadas en ella en caso concreto del diagnóstico por imagen del cáncer de próstata, aunque no debe obviarse al plantear la estrategia de la biopsias prostáticas.

\section{RENTABILIDAD PROFESIONAL Y SOCIAL}

El campo de aplicación de la ecografía urológica abarca todo el aparato urinario y genital masculino. Su capacidad para ofrecer las informaciones esenciales de forma inocua, rápida y sencilla la convierten en una de las técnicas de imagen de mayor eficacia y difusión.

La ecografía es capaz de ofrecer las respuestas que todo urólogo se plantea ante un paciente, después de realizar la correspondiente historia clínica y exploración. Su accesibilidad y alta sensibilidad hacen que figure en el inicio de los algoritmos diagnósticos de una gran mayoría de patologías urológicas donde se precisan pruebas de alta sensibilidad al inicio de las exploraciones complementarias.

El rendimiento de las exploraciones ecográficas depende de la experiencia del operador en el momento de adquirir e interpretar las imágenes como al correlacionarlas con las posibilidades patológicas de cada cuadro clínico y de cada órgano explorado. Igualmente depende del uso de la tecnología adecuada. A pesar de su aparente simplicidad la ecografía 
exige una adecuada formación y experiencia para garantizar la realización de un examen ecográfico correcto, ya que en el caso de exámenes ecográficos defectuosos puede llevar a resultados falsamente positivos o negativos, de nefastas consecuencias para el paciente. No hay que olvidar que en la secuencia del acto médico, cada exploración debe aportar los hallazgos que se realizan en su óptimo nivel de calidad. Los exámenes defectuosos dan resultados inaceptables para el profesional y para el paciente, con la consiguiente responsabilidad para el médico. $\mathrm{Si}$ toda técnica urológica conlleva la necesidad de una buena formación, para practicarla con garantías, la ecografía todavía más.

La ecografía tiene un enorme valor en la asistencia clínica diaria porque mejora la capacidad resolutiva del urólogo en tres aspectos:

- En el diagnóstico: es una exploración de alta sensibilidad y especificidad, fiable, inocua, accesible y económica. Con la ventaja de que puede repetirse todas las veces que se crea conveniente.

\section{TABLA I. RENTABIIIDAD DIAGNÓSTICA DE LA ECOGRAFÍA}

\begin{tabular}{|c|c|c|c|}
\hline EXPLORACIÓN & TÉCNICA & ÍNDICE & REFERENCIA \\
\hline Hidronefrosis & $\begin{array}{l}\text { Ecografía } \\
\text { externa }\end{array}$ & $\begin{array}{l}\text { Sensibilidad: } 90-100 \% \\
\text { Especificidad: } 90-98 \%\end{array}$ & Pollack H.M. y cols. (4) \\
\hline Masas renales & $\begin{array}{l}\text { Ecografía } \\
\text { externa }\end{array}$ & Exactitud: $98 \%$ & Talner L.B. y cols. (5) \\
\hline $\begin{array}{l}\text { Diferencia entre masas rena- } \\
\text { les vascularizadas o no }\end{array}$ & Doppler color & Sensibilidad: $100 \%$ & Spahn M. y cols. (6) \\
\hline $\begin{array}{l}\text { Detección de litiasis en pa- } \\
\text { cientes con cólico nefrítico }\end{array}$ & $\begin{array}{l}\text { Ecografía } \\
\text { externa }\end{array}$ & $\begin{array}{l}\text { Sensibilidad: } 93 \% \\
\text { Especificidad: } 95 \%\end{array}$ & Patlas M. y cols. (7) \\
\hline $\begin{array}{l}\text { Detección de patología uroló- } \\
\text { gica en Urgencias }\end{array}$ & $\begin{array}{l}\text { Ecografía } \\
\text { externa }\end{array}$ & $\begin{array}{l}\text { Sensibilidad: } 84 \% \\
\text { Especificidad: } 97 \%\end{array}$ & Nargund V.H. y cols. (8) \\
\hline Medida del residuo vesical & $\begin{array}{l}\text { Ecografía } \\
\text { externa }\end{array}$ & $\begin{array}{l}\text { Índice de correlación: } 0,86 \\
-0,98\end{array}$ & Corabian P. (9) \\
\hline $\begin{array}{l}\text { Detección de tumores vesi- } \\
\text { cales }\end{array}$ & $\begin{array}{l}\text { Ecografía } \\
\text { externa }\end{array}$ & $\begin{array}{l}\text { Exactitud: } \\
\text { <5mm.: } 38 \% \\
5-10 \mathrm{~mm} .: 82 \% \\
>10 \mathrm{~mm}: 100 \%\end{array}$ & Malone P.R. (10) \\
\hline $\begin{array}{l}\text { Detección del cáncer de } \\
\text { próstata } \\
\text { (post T.R. y PSA) }\end{array}$ & $\begin{array}{l}\text { Ecografía } \\
\text { transrectal }\end{array}$ & $\begin{array}{l}\text { Sensibilidad: } 84 \% \\
\text { Especificidad: } 67 \%\end{array}$ & Aarnink R.G. y cols. (11) \\
\hline $\begin{array}{l}\text { Detección de recidiva del } \\
\text { cáncer de próstata post-pros- } \\
\text { tatectomía }\end{array}$ & Doppler color & $\begin{array}{l}\text { Sensibilidad: } 86 \% \\
\text { Especificidad: } 100 \%\end{array}$ & Sudakoff G.S. y cols. (12) \\
\hline $\begin{array}{l}\text { Masas escrotales intratesticu- } \\
\text { lar o extratesticular }\end{array}$ & $\begin{array}{l}\text { Ecografía } \\
\text { externa }\end{array}$ & Exactitud: $100 \%$ & Coret A. y cols. (13) \\
\hline $\begin{array}{l}\text { Detección de tumores intrates- } \\
\text { ticulares }\end{array}$ & $\begin{array}{l}\text { Ecografía } \\
\text { externa }\end{array}$ & Sensibilidad: $100 \%$ & Gallardo E. y cols. (14) \\
\hline
\end{tabular}


- En el seguimiento de los pacientes: la exploración puede ser repetida todas las veces que se quiera y comparar las imágenes con los datos anteriores. Además suministra información sobre la evolución y morfología del órgano estudiado, especialmente en las patologías urológicas más frecuentes como son la $\mathrm{HBP}$, cólicos nefríticos, hidronefrosis y tumores vesicales.

- En el tratamiento: permite la colocación de nefrostomías percutáneas, mediante la punción ecodirigida que resulta de gran sencillez. Igualmente permite la punción y evacuación de quistes renales o colecciones perirrenales. Es el instrumento imprescindible en la realización de biopsias prostáticas ecodirigidas, ya sea por vía transrectal o transperineal. También nos permite la punción de abscesos prostáticos por vía transperineal.

En su manejo, el urólogo es el profesional más capacitado para sacarle su máximo rendimiento ya que está en la mejor posición para confrontar los hallazgos de la imagen con la historia clínica y la exploración física, situando la imagen en el adecuado contexto de la clínica. Esta confrontación de la imagen con la clínica es el valor añadido que ofrece la ecografía urológica puesta en manos del urólogo. Hace años que nuestro grupo defendemos un concepto que denominamos Ecografía Urológica Integral (15), que consiste en que el urólogo disponga en su consulta y sala de exploraciones de un ecógrafo universal apto para ecografía externa y transrectal. Con este instrumento el urólogo obtiene en le mismo acto de la consulta un diagnóstico ecográfico-morfológico del aparato urinario y genital que en muchas ocasiones es suficiente para establecer un diagnostico y establecer inmediatamente una pauta de tratamiento. Mediante la Ecografía Urológica Integral como concepto y método de exploración, podemos conocer el estado del aparato urinario superior e inferior, o del aparato genital en un breve periodo de tiempo de 5 a 10 minutos, resultando la primera prueba complementaria esencial para completar la hipótesis diagnóstica. Esta accesibilidad de la ecografía y su versatilidad la sitúan como el complemento perfecto para las Consultas de Alta Resolución, donde el paciente acude con una sospecha diagnóstica o un cuadro sintomático y en el mismo acto médico se realiza la historia clínica, la exploración y todas las pruebas complementarias, finalizando la consulta con el diagnóstico del paciente y la orientación terapéutica.

El manejo directo del ecógrafo por parte del urólogo tiene varias consecuencias:

-La realización de ecografías por parte del Servicio de Urología, racionaliza y limita la deman- da de ecografías hasta ahora solicitadas a los Servicios de Radiología

-Disminuye de forma importante la petición de exploraciones radiológicas, como urografías y T.C.

-Disminución de cistoscopias y otras exploraciones agresivas. La combinación de ecografía y citología, o ecografía y el test NMP22 BladderCheck, permite seguir la evolución de los tumores vesicales en pacientes sometidos a RTU.

-Un aumento de la eficacia y eficiencia asistencial al disminuir el numero de pruebas, número de revisiones, y favorecer la consultas de alta resolución.

Tradicionalmente los Servicios de Urología son grandes demandantes de ecografías diagnósticas al Servicio de Radiología, por lo que la dotación de ecógrafos en los Servicios de Urología podría cubrir todas las necesidades de exploraciones ecográficas que realizarían los propios urólogos.

Sin embargo existe una gran variabilidad en cuanto a la dotación de equipos de ecografía entre unos Servicios de Urología y otros y en cuanto al uso y rendimiento que los profesionales hacen de ellos. Hay profesionales que defienden que todos los facultativos de un Servicio de Urología deberían estar formados en ecografía y disponer de un ecógrafo en todas las consultas y quirófano. Sin embargo en la mayor parte de los Servicios de Urología españoles solo se dispone de un ecógrafo por Servicio que es destinado a la Sala de Exploraciones Especiales y quirófano, por lo cual la inmensa mayoría de las exploraciones ecográficas demandadas por los urólogos siguen siendo realizadas por los Servicios Centrales de Radiodiagnóstico. El problema es que no existe un consenso o protocolo sobre el equipamiento mínimo de ecógrafos en los Servicio de Urología, ni sobre el tiempo mínimo que debe dedicarse a cada estudio. Esta variabilidad en la implantación de la ecografía en los Servicios de Urología españoles refleja diversos problemas subyacentes de la Sanidad Española, como son la insuficiencia presupuestaria para la dotación y actualización tecnológica de los hospitales, la resistencia de los Servicios de Radiología a perder la ecografía urológica, o la diferente implicación de los urólogos españoles en esta técnica.

Además de reflejar los problemas subyacentes, también existen distintos puntos de vista de lo que debe ser la ecografía diagnóstica en Urología:

- Los que piensan que los ecógrafos deben de estar únicamente en los Servicios de Radiodiagnóstico. 
- Los que siguen un criterio estructural y consideran al ecógrafo como un instrumento complementario de uso limitado a solo unas escasas patologías y encaminado a las actuaciones de ecografía intervencionista, por lo cual estiman que la dotación de ecógrafos debe reducirse a uno por Servicio.

- Los partidarios de seguir un criterio funcional, como nosotros, que contemplamos el ecógrafo como un instrumento indispensable, como son los guantes de tacto o el fonendoscopio, considerando que los ecógrafos deben de estar disponibles en consulta, planta de hospitalización y en quirófano, es decir allí donde hay un paciente urológico. En este caso el número de ecógrafos debe ser múltiple en función del volumen del Servicio y de la población atendida.

No existen datos de libre acceso publicados por las Consejerías de Sanidad de las distintas Comunidades Autónomas que nos permitan analizar y comparar el grado de implantación y rendimientos de la ecografía en los Servicios de Urología. A través de contactos personales y alguna publicación como la del Instituto Galego de Medicina Técnica (16) podemos formarnos una idea aproximada del estado de esta cuestión.

La mayor implantación de la ecografía urológica en España se ha realizado en sector privado donde el ecógrafo es un accesorio indispensable en consultas, sala de exploraciones especiales y quirófano. Muchos urólogos españoles, con ejercicio profesional privado o mixto, confiesan que el ecógrafo se ha convertido en un instrumento indispensable en su consulta y consideran inimaginable volver al modelo de consulta clásica donde las primeras exploraciones por imagen se solicitaban al Servicio de Radiología. En el sector público la implantación es totalmente dispar, reflejando las tres tendencias anteriormente expuestas, aunque la mayoría de los Servicios de Urología disponen al menos de un ecógrafo por Servicio, todavía estamos muy lejos de la implantación de los ecógrafos en las consultas de urología de forma generalizada.

En la siguiente Tabla (Tabla II) presentamos la estadística de nuestro Servicio, perteneciente a un hospital de área, nivel II, que consideramos que es uno de los hospitales que mayor utilización hacen de le ecografía urológica. En nuestro Servicio la implantación de la ecografía se ha realizado siguiendo un criterio funcional. Aprovechando al máximo la versatilidad y movilidad de los ecógrafos hemos conseguido que siempre haya un ecógrafo donde el urólogo lo necesite. Las ecografías demandadas por el Servicio de Urología las realiza el propio Servicio, ya sean ecografías diagnósticas o intervencionistas. El servicio de Radiología realiza únicamente las ecografías solicitadas por el Servicio de Urgencias $u$ otras especialidades.

TABLA II. UTILIZACIÓN Y RENDIMENTO DE LA ECOGRAFÍA EN EL HOSPITAL SANTA BARBARA-SORIA

\begin{tabular}{|l|l|l|}
\hline & $\mathbf{2 0 0 3}$ & $\mathbf{2 0 0 4}$ \\
\cline { 2 - 3 } TOTAL DE ECOGRAFÍAS & 5.784 & 5.545 \\
\hline Ecografías renales & 1.525 & 1.390 \\
\hline Ecografías vesicales & 3.721 & 3.672 \\
\hline Ecografías escrotales & 258 & 185 \\
\hline Ecografías transrectales & 280 & 298 \\
\hline Total de ecografías/1000 habitantes & 64,6 & 61,9 \\
\hline Ecógrafos existentes en el Servicio de Urología & 2 & 2 \\
\hline Facultativos /ecógrafos & 2,5 & 2,5 \\
\hline Total número de consultas & 7.673 & 7.534 \\
\hline Consultas/1000 habitantes & 85,7 & 84,1 \\
\hline Ecografía/consulta & 0,75 & 0,73 \\
\hline
\end{tabular}




\section{RENTABILIDAD ECONÓMICA}

Tal vez sea porque sus ventajas son evidentes a simple vista, apenas existen estudios basados en evidencias científicas sobre la rentabilidad de la ecografía en el campo de la urología. Podemos encontrar algún artículo de opinión pero no existen ensayos clínicos controlados que comparen desde el punto de vista de rentabilidad económica, las distintas técnicas de imagen utilizadas en el diagnóstico urológico.

Todos los especialistas familiarizados con la ecografía tenemos la experiencia y convencimiento de que los estudios ecográficos son más económicos que otras técnicas, pero en nuestro país no existen estudios de evaluación económica dirigidos a evaluar el coste de la ecografía comparada con otros procedimientos. Si recurrimos a la Base de datos de Evaluación Económica del NHS ( The NHS Economic Evaluation Database- NHS EED) (17) encontramos algunas referencias que confirman nuestra primera impresión. La NHS EED es producida por el Centre for Reviews and Dissemination( NHS CRD) en la Universidad de York (Gran Bretaña).

El NHS CRD define la evaluación económica como una de las tres opciones siguientes:

a- Un análisis coste-beneficio, que mide tanto el coste como los beneficios en términos económicos y calcula los beneficios económicos netos o las perdidas.

b- Un análisis coste-efectividad que compara intervenciones con un resultado común para descubrir cual de ellas produce el máximo resultado para la misma aportación de recursos en una determinada población.

c- Un análisis coste-utilidad, que mide los beneficios de tratamiento o tipos de atención alternativos recurriendo a medidas de utilidad claramente definidas, como los Años de Vida Ajustados por Calidad.

En la base de datos de NHS CRD hemos encontrado algunos estudios de tipo coste-efectividad relacionados con el uso de la ecografía en el campo de la urología, aunque y que señalar que son muy escasos e incompletos.

Un estudio de análisis coste-efectividad destinado a evaluar las masas renales detectadas por urografía, comparando la ecografía y TC (18) concluye que la sonografía es el método mejor en dicha comparación, debiendo reservarse el TC para un número delimitado y específico de indicaciones.
Sin embargo en otro estudio de análisis coste-efectividad para evaluar el uso de la ecografía utilizada de forma sistemática en la fase temprana de los cólicos renales (19), el uso de la ecografía incrementaba los costes del diagnóstico, pero los resultados de este estudio deben ser tratados con precaución porque existía un alto porcentaje de falsos positivos para la ecografía en la fase temprana del cólico nefrítico, y el análisis económico estaba basado exclusivamente en los costes.

En otro estudio (20) de análisis de costes en pacientes necesitados de cateterismo vesical en el post-operatorio se realizó una comparación entre la utilización de ecógrafos portátiles para la determinación del volumen vesical en comparación con el cateterismo vesical intermitente. Aunque no hubo una diferencia estadísticamente significativa respecto a los episodios de infección urinaria entre el grupo en que se realizó ecografía vesical y el grupo de cateterismos, se apreciaba una tendencia menor hacia las infecciones en el grupo en que se utilizó la ecografía para la medida del volumen residual vesical. El uso de ecógrafos portátiles para medir el volumen de orina residual evita los cateterismos innecesarios. Sin embargo el análisis económico es defectuoso para sacar conclusiones de tipo económico sobre costebeneficio, comparando estos dos métodos. El estudio concluye que la ecografía es precisa, disminuye el número de cateterismos, reduce los costes de tiempo y provee una alta satisfacción al paciente.

En todas las bases de datos aparecen diversos estudios que se enuncian con análisis de coste-efectividad, pero la realidad es que no son evaluaciones económicas y mucho menos un análisis de coste-beneficio comparando la ecografía con otras alternativas. No existe ningún estudio de costes que compare la ecografía realizada por urólogos en sus consultas, con el sistema tradicional de ecografías solicitadas desde consulta a los Servicios de Radiología. Todo ello nos hace concluir que es necesaria la realización de estudios de evaluación que incluya todos los costes económicos además de los beneficios de salud para el paciente y la comunidad.

\section{BIBLIOGRAFIA y LECTURAS RECOMENDADAS (*lectura de interés $y^{* *}$ lectura fundamental)}

**1. PASSAS MARTINEZ, J.; RODRIGUEZ ANTOLIN, A.; DE LA ROSA, S.: "Ecografía en Urología”. Actas Urol. Esp., 26: 650, 2002.

**2. MCACHRAN, S.E.; DOGRA, V.; RESNICK, M.I.: "Office urologic ultrasound". Urol. Clin. North Am., 32: 337, 2005. 
**3. RODRÍGUEZ PATRÓN, R.; MAYAYO, T.; ALONSO, M. y cols.: "Resultados de una serie de 6.000 pacientes con biopsia transrectal ecodirigida de próstata. Parte I. Anatomía patológica, tacto rectal, ecografía transrectal y PSA". Arch. Esp. Urol., 58: 611, 2005.

4. POLLACK, H.M.; BANNER, M.P.; ARGER, P.H. y cols.: "The accuracy of gray-scale renal ultrasonography in differentiating cystic neoplasms from benign cysts". Radiology., 143: 741, 1982.

5. TALNER, L.B.; SCHEIBLE, W.; ELLENBOGEN, P.H. y cols.: "How accurate is ultrasonography in detecting hydronephrosis in azotemic patients?". Urol. Radiol., 3: 1, 1981.

6. SPAHN, M.; PORTILLO, F.J.; MICHEL, M.S. y cols.: "Color Duplex sonography vs. computed tomography: accuracy in the preoperative evaluation of renal cell carcinoma". Eur. Urol., 40: 337, 2001.

7. PATLAS, M.; FARKAS, A.; FISHER, D. y cols.: "Ultrasound vs CT for the detection of ureteric stones in patients with renal colic". Br. J. Radiol., 74: 901, 2001.

8. NARGUND, V.H.; CUMMING, J.A.; JERWOOD, D. y cols.: "Ultrasound in urological emergency: results of self audit and implications for training". Int. Urol. Nephrol., 28: 267, 1996.

*9. CORABIAN, P.: "Bladder ultrasound scanning for the measurement - technology assessment report. Bladder ultrasound scanning for the measurement of post-void residual urine volume". The Health Technology Assessment Unit. Alberta Heritage Foundation for Medical Research.

http://www.ahfmr.ab.ca/hta/hta-publications/briefs/Bladder.1.php

10. MALONE, P.R.: "Transabdominal ultrasound surveillance for bladder cancer". Urol. Clin. North Am., 16: 823, 1989.

11. AARNINK, R.G.; BEERLAGE H.P.; DE LA ROSETTE J.J. y cols.: "Transrectal ultrasound of the prostate: innovations and future applications". J. Urol., 159: 1568, 1998.
12. SUDAKOFF, G.S.; SMITH, R.; VOGELZANG, N.J. y cols.: "Color Doppler imaging and transrectal sonography of the prostatic fossa after radical prostatectomy: early experience". AJR Am. J. Roentgenol., 167: 883, 1996.

13. CORET, A.; LEIBOVITCH, I.; HEYMAN, Z. y cols.: "Ultrasonographic evaluation and clinical correlation of intratesticular lesions: a series of 39 cases". Br. J. Urol., 76: 216, 1995.

*14. GALLARDO AGROMAYOR, E.; PEÑA-GOMEZ, E.; LOPEZ RASINES, G.; y cols.: "Tumores testiculares. Hallazgos ecográficos." Arch. Esp. Urol., 49: 622, 1996.

15. ESPUELA ORGAZ, R.; ABAD MENOR, F.; MARTINEZ PEREZ, E. y cols.: "Ecografía urológica integral. I: Patología renoureteral". Actas Urol. Esp., 2: 119, 1988.

*16. RICCI VOLTAS, C.; VILLAR BARREIRO, J.; SANCHEZ VARELA, M.: "Criterios para la dotación de ecógrafos en los servicios de ginecología y obstetricia, digestivo, cirugía vascular, cardiología, urología y oftalmología”. Rev. Calidad Asistencial, 18: 145, 2003.

*17. NHS ECONOMIC EVALUATION DATABASE (NHS EED).: http://www.york.ac.uk/inst/crd/nhsdhp.htm.

18. EINSTEIN, D.M.; HERTS, B.R.; WEAVER. R. y cols.: "Evaluation of renal masses detected by excretory urography: cost-effectiveness of sonography versus CT". AJR Am. J. Roentgenol., 164: 371, 1995.

19. GIMONDO, P.; MASTROPASQUA, G.; CREMONA, A. y cols.: "Early and systematic use of ultrasonography in emergency patients with renal colic: analysis of the actual diagnostic efficacy". Radiol. Med. (Torino)., 92: 421, 1996.

20. FREDERICKSON, M.; NEITZEL, J.J.; MILLER, E.H. y cols.: "The implementation of bedside bladder ultrasound technology: effects on patient and cost postoperative outcomes in tertiary care". Orthop. Nurs., 19: 79, 2000. 